\title{
Desempeño térmico de un espacio habitable con ventilación modo mixto. Diferentes condiciones volumétricas y diferentes condiciones térmico ambientales en el Estado de Guanajuato. México
}

\section{Thermal performance of a living space with mixed mode ventilation. Different volumetric conditions and different thermal-environmental conditions in the State of Guanajuato. Mexico}

\author{
VÁZQUEZ-TORRES, Claudia Eréndira †*, GÓMEZ-AMADOR, Adolfo y ESCOBAR-DEL POZO, \\ Carlos
}

Universidad de Colima

ID $1^{\mathrm{er}}$ Autor: Claudia Eréndira, Vázquez-Torres / ORC ID: 0000-0002-5388-0780, CVU CONACYT ID: 181807

ID $1^{\text {er }}$ Coautor: Adolfo, Gómez-Amador / ORC ID: 0000-0002-1071-0861, CVU CONACYT ID: 26110

ID $2^{\text {do }}$ Coautor: Carlos, Escobar-Del Pozo / ORC ID: 0000-0002-8732-8791, Researcher ID Thomson: G-8111-2018, CVU CONACYT ID: 43347

DOI: $10.35429 / J A D .2019 .10 .3 .11 .19$

Recibido 23 de Octubre, 2018; Aceptado 17 de Diciembre, 2018

\begin{abstract}
Resumen
En el presente trabajo se muestra el desempeño térmico de un espacio habitable con ventilación modo mixto. Espacio localizado en una casa representativa del registro de vivienda por tipología y por superficie (Comisión Nacional de Vivienda, 2017). Se presentan resultados de temperatura operativa para un caso base y seis modelos de evaluación virtual (MEVi) variando el volumen de aire interior, empleando la herramienta de cálculo Design Builder®. El objetivo principal consistió en determinar, el modelo con menor uso de sistemas activos al variar el volumen de aire interior con un rango de confort determinado, de acuerdo a cada condición térmica, al usar ventilación modo mixto. Resultados clave: A) Se determinaron necesidades de calentamiento y enfriamiento en tres condiciones térmicas, B) al incrementar el volumen del aire interior, se presentó una disminución en las horas de confort por año, y una clara tendencia a mantener la ventana abierta para las tres condiciones térmicas, C) se demuestra que, en las condiciones térmicas: frío sub húmedo, cálido sub húmedo y templado sub húmedo, que se presentan en el estado de Guanajuato México, el volumen de aire interior es directamente proporcional a la demanda energética por año.
\end{abstract}

Ventilación modo mixto, Desempeño térmico, Condiciones climáticas.

\begin{abstract}
In the present work the thermal performance of a habitable space with mixed mode ventilation is shown. Space located in a dwelling representative of the housing register by type and surface, data from CONAVI (2017). Results of operating temperature for a base case and six virtual evaluation models (MEVi) are presented, varying the interior air volume, using Design Builder ${ }^{\circledR}$ calculation tool. The main objective was to determine the model with less use of active systems by varying the volume of indoor air with a certain comfort range, according to each thermal condition, when using mixed mode ventilation. Key results: A) Heating and cooling needs were determined in three thermal conditions, B) by increasing the volume of indoor air, there was a decrease in comfort hours per year, and a clear tendency to keep the window open for three thermal conditions, C) it is demonstrated that, in the thermal conditions: cold sub humid, warm sub humid and temperate sub humid, that occur in the state of Guanajuato Mexico, the volume of indoor air is directly proportional to the energy demand by year.
\end{abstract}

Citación: VÁZQUEZ-TORRES, Claudia Eréndira, GÓMEZ-AMADOR, Adolfo y ESCOBAR-DEL POZO, Carlos. Desempeño térmico de un espacio habitable con ventilación modo mixto. Diferentes condiciones volumétricas y diferentes condiciones térmico ambientales en el Estado de Guanajuato. México. Revista de Arquitectura y Diseño. 2019, 3-10: 11-19

\footnotetext{
*Correspondencia al Autor (Correo Electrónico: claudiaerendira@gmail.com)

$\dagger$ Investigador contribuyendo como primer autor.
} 


\section{Introducción}

El auge de los nuevos desarrollos de vivienda implica el uso y reproducción de las mismas dimensiones mínimas de los espacios arquitectónicos en diferentes condiciones climáticas. Para obtener una vivienda calificada como económica o popular, se debe percibir menos de cuatro salarios mínimos (5.1 USD), por lo que, difícilmente se invierte en un mejoramiento de las condiciones del espacio.

Lo anterior nos lleva a cuestionar, si se utiliza el mismo modelo de vivienda para satisfacer la demanda habitacional utilizando la misma distribución y dimensión de espacios en condiciones climáticas diferentes, el desempeño térmico podría variar significativamente y generar condiciones térmicamente ineficientes en sus espacios interiores y con baja probabilidad por parte de los usuarios para invertir en el mejoramiento del desempeño térmico de sus viviendas.

El enfoque en la evaluación del rendimiento térmico ha sido en paredes o techos de edificios envolventes. El análisis dependiente del tiempo de la transferencia de calor ha sido a través de una pared / techo destinado a estudiar el efecto de los materiales, sin considerar las dimensiones del espacio habitable.

En este caso, se determina como variable independiente al volumen del aire interior para conocer la demanda de energía, y las tareas de calentamiento, enfriamiento y confort que se presentaron en tres diferentes condiciones térmicas del Estado de Guanajuato, México. La metodología fue dividida en 12 partes (Tabla 01).

Para la realización de este estudio, se seleccionó el modelo de vivienda utilizado con mayor frecuencia en el estado de Guanajuato, su tipología, materiales y sistemas constructivos; para comparar en diferentes modelos de evaluación virtual (MEVi), el desempeño térmico en las condiciones térmicas: templado sub húmedo, cálido sub húmedo y frío sub húmedo.
Se determinó como herramienta de cálculo el programa de análisis de energía DesignBuilder $®$, con el objetivo de evaluar el rendimiento energético y obtener valores cuantitativos para identificar el modelo donde se utiliza en menor medida los sistemas activos con un rango de confort determinado que nos permita mejorar las condiciones de confort térmico de acuerdo a las condiciones climáticas propuestas cuando utilizamos ventilación modo mixto.

\begin{tabular}{|c|c|c|}
\hline No. & Método & Metodología \\
\hline 1 & $\begin{array}{l}\text { Selección unidad de } \\
\text { estudio }\end{array}$ & $\begin{array}{l}\text { Estadísticas Comisión } \\
\text { Nacional de la } \\
\text { Vivienda, lineamientos } \\
\text { INFONAVIT }\end{array}$ \\
\hline 2 & $\begin{array}{l}\text { Obtención de datos } \\
\text { climatológicos } \\
\text { exteriores }\end{array}$ & $\begin{array}{l}\text { Normales } \\
\text { Climatológicas del } \\
\text { Servicio Meteorológico } \\
\text { Nacional }\end{array}$ \\
\hline & & Meteonorm® \\
\hline 3 & $\begin{array}{l}\text { Limitaciones normativas } \\
\text { locales }\end{array}$ & $\begin{array}{l}\text { Reglamentación vigente } \\
\text { de localidades: León, } \\
\text { San Felipe y Xichú Gto. }\end{array}$ \\
\hline 4 & $\begin{array}{l}\begin{array}{l}\text { Propiedades } \\
\text { higrotérmicas de los } \\
\text { materiales }\end{array} \\
\end{array}$ & $\begin{array}{l}\text { Análisis de diferentes } \\
\text { autores }\end{array}$ \\
\hline 5 & \begin{tabular}{lll} 
Diagnóstico & \multicolumn{2}{c}{ climático. } \\
Horas de confort y & con de \\
disconfort. & Rango de \\
confort & &
\end{tabular} & $\begin{array}{l}\text { Climate Consultant, } \\
\text { Luna (2017), Gómez } \\
(2018)\end{array}$ \\
\hline 6 & $\begin{array}{l}\text { Estrategia de ventilación } \\
\text { modo mixto }\end{array}$ & $\begin{array}{l}\text { Análisis de diferentes } \\
\text { autores }\end{array}$ \\
\hline 7 & Condiciones de frontera & $\begin{array}{lll}\begin{array}{l}\text { Análisis } \\
\text { autores }\end{array} & \text { diferentes } \\
\end{array}$ \\
\hline 8 & on & Design Builder ${ }^{\circledR}$ \\
\hline 9 & & \\
\hline 10 & $\begin{array}{l}\text { Manipulación del } \\
\text { volumen de aire interior }\end{array}$ & Design Builder® \\
\hline 11 & $\begin{array}{l}\text { Matriz de resultados de } \\
\text { simulación }\end{array}$ & SPSS ${ }^{\circ}$, Excel \\
\hline 12 & Análisis de resultados & \\
\hline
\end{tabular}

Tabla 1 Método y metodología utilizados en la investigación

\section{Descripción del modelo}

La unidad de estudio es una vivienda de un solo nivel, de estancia-comedor, cocina y patio de servicio y dos recámaras; en un área de $39.78 \mathrm{~m}^{2}$. El sistema constructivo consiste en muros de block de concreto y losa de concreto (figura 01). 


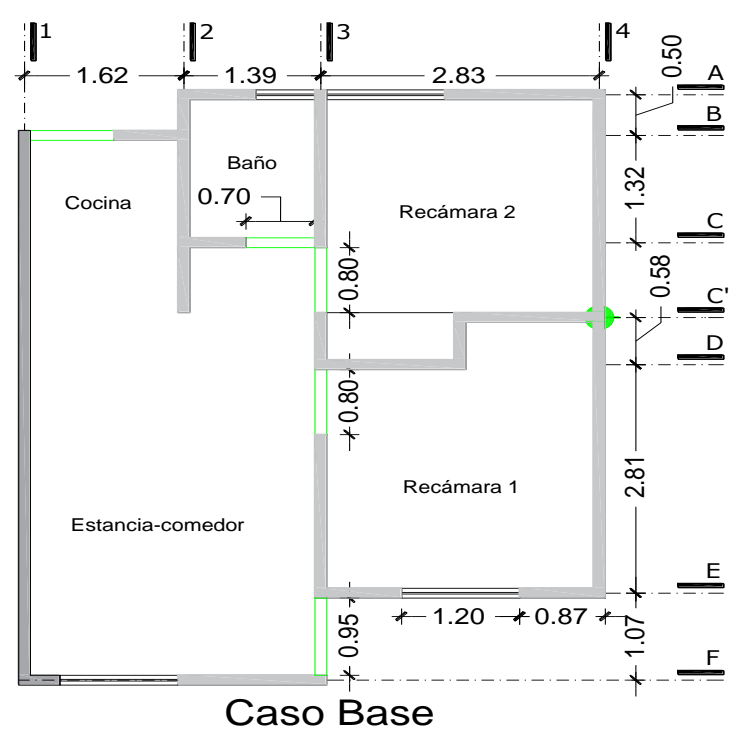

Figura 1 Unidad de estudio

La altura interior del caso base es, de acuerdo a la normatividad vigente, de $2.3 \mathrm{~m}$. Se seleccionó la recámara 1 como espacio representativo, por ser el espacio en la vivienda donde se permanecen más horas destinadas al descanso.

\section{Diseño de la simulación}

El diseño de la simulación constó de cuatro fases: escenario de la simulación, selección del modelo de confort, simulación y Resultados (Figura 02). Por cada condición térmica y por cada modelo se realizaron dos simulaciones (con ventanas abiertas y con ventanas cerradas). Lo que produjo dos resultados por cada hora, a partir de estos resultados, se realizó una operación selectiva, ésta corresponde a la acción que puede realizar el habitante de abrir o cerrar la ventana en función de un rango de confort determinado; y que en las simulaciones realizadas consistió en la elección de la temperatura operativa, con base horario, que permaneció o se acercó más al rango de confort determinado (Figura 03)

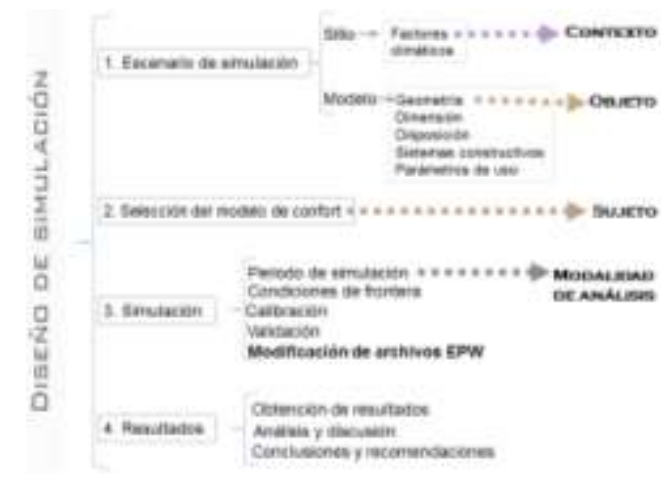

Figura 2 Diseño de la simulación

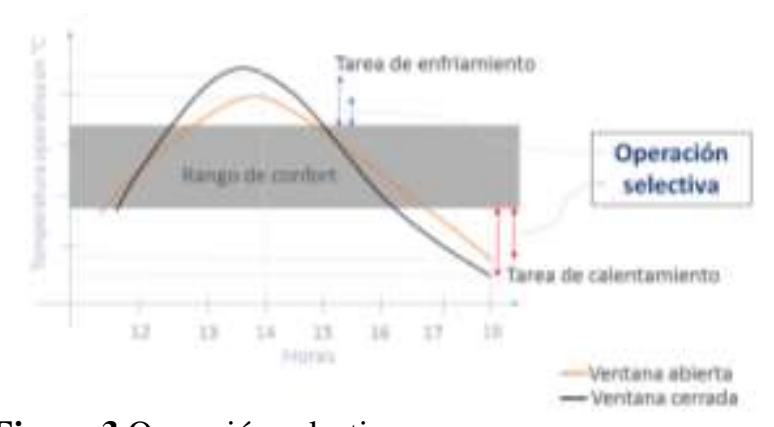

Figura 3 Operación selectiva

1. Modelo en Design Builder. El modelo geométrico corresponde a la figura 04 , donde se muestra la ubicación del espacio representativo seleccionado (Recámara 1).
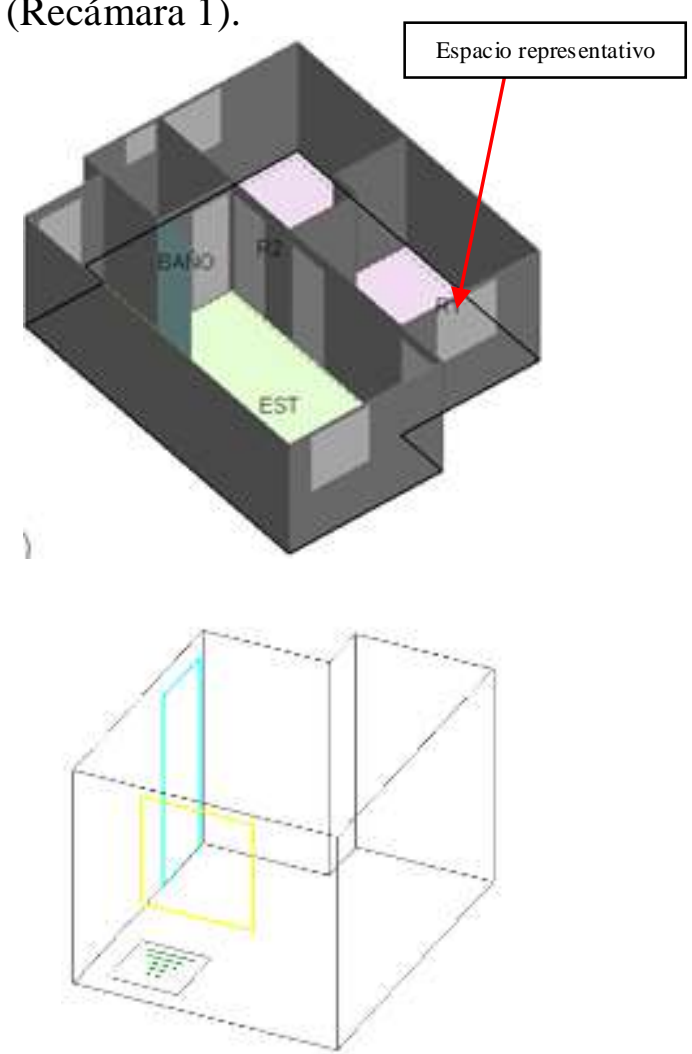

Figura 4 Modelo geométrico. La puerta del espacio representativo tiene dimensiones $0.80 \times 2.10 \mathrm{~m}$, y la ventana V1 $1.20 \mathrm{X} 1.20 \mathrm{~m}$. Por funcionamiento de los espacios, al realizar las simulaciones, se conservaron las dimensiones de puerta y ancho de closet, mientras que la ventana se incrementó en la misma proporción del volumen de aire interior para garantizar el porcentaje de iluminación y ventilación establecido originalmente.

2. Proporción de crecimiento. La variación del volumen de aire interior se fundamenta en los siguientes aspectos: 1) en el reglamento para el municipio de San José Iturbide, donde se ubica el caso base, y al no contener medidas mínimas reglamentarias, se determina que la altura del caso base, es representativa de la altura mínima del espacio habitable interior que corresponde a $2.3 \mathrm{~m}$. 
Por lo que a partir de esta medida se realizaron los Módulos de Evaluación Virtual (MEVi). La dimensión mínima se incrementó de acuerdo a la proporción mostrada en la Tabla 02.

\begin{tabular}{|l|r|r|}
\hline \multicolumn{1}{|c|}{ Modelo } & Proporción & \multicolumn{1}{c|}{ Altura interior } \\
\hline Caso Base & 1.00 & 2.30 \\
\hline MEVi 1 & 1.25 & 2.88 \\
\hline MEVi 2 & 1.50 & 3.45 \\
\hline MEVi 3 & 1.75 & 4.03 \\
\hline MEVi 4 & 2.00 & 4.60 \\
\hline MEVi 5 & 2.25 & 5.18 \\
\hline MEVi 6 & 2.50 & 5.75 \\
\hline
\end{tabular}

Tabla 2 Proporción de crecimiento

2) El punto desde el cual se realizó el incremento del volumen del aire interior es el eje 4-C', conservando dimensiones de puertas, ancho en muros $(12 \mathrm{~cm})$ y closets, debido a la funcionalidad de los espacios (Figura 05).

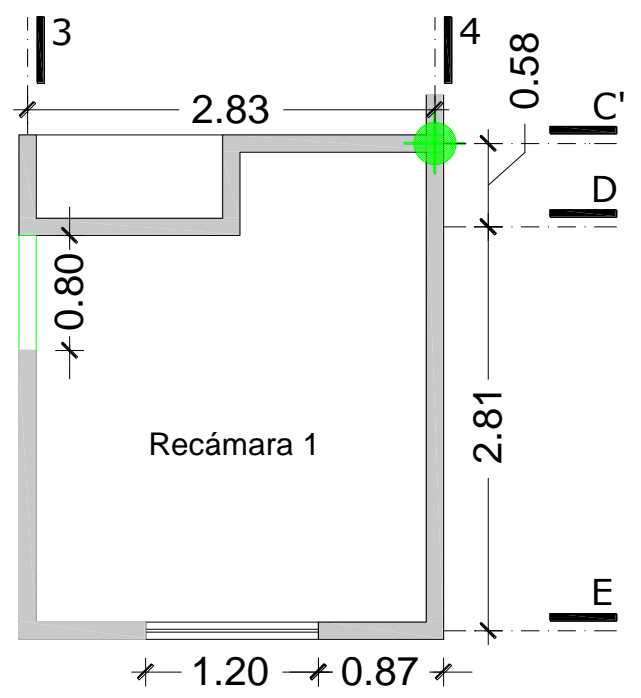

Figura 5 Representación de la referencia de crecimiento progresivo para crear los MEVi a partir del caso base

3) Con estos datos, se obtuvieron las condiciones de frontera para el Objeto de Estudio (OE) que corresponde a la vivienda completa, y para el espacio representativo (R1) que corresponde a la Recámara 1, como lo indica la siguiente Tabla:

\begin{tabular}{|c|c|c|c|c|c|c|c|}
\hline \multirow{2}{*}{\multicolumn{2}{|c|}{ Referencia | }} & \multirow{2}{*}{$\begin{array}{c}\text { Propor } \\
\text { ción }\end{array}$} & \multirow{2}{*}{$\begin{array}{c}\text { Altura } \\
\text { interior }\end{array}$} & \multicolumn{2}{|c|}{$\begin{array}{l}\text { Volumen } \\
\text { del aire } \\
\text { interior }\end{array}$} & \multicolumn{2}{|c|}{ Actividad } \\
\hline & & & & & & & \\
\hline \multirow{2}{*}{$\begin{array}{l}\text { Caso } \\
\text { Base }\end{array}$} & $\mathrm{OF}$ & \multirow[b]{2}{*}{1} & \multirow{2}{*}{2.30} & 39.7 & \multirow{2}{*}{$\begin{array}{c}18.4 \\
9\end{array}$} & 005 & 1508 \\
\hline & $\mathrm{R} 1$ & & & $\frac{8}{804}$ & & 025 & 7163 \\
\hline \multirow{3}{*}{ MEVil } & $\mathrm{OF}$ & \multirow{3}{*}{1.25} & \multirow{3}{*}{2.88} & 61.6 & \multirow{3}{*}{$\begin{array}{c}37.4 \\
9\end{array}$} & 003 & 073 \\
\hline & UL & & & 7 & & 0.00 & 9.13 \\
\hline & $\mathrm{R} 1$ & & & $\begin{array}{c}13.0 \\
4\end{array}$ & & 0.15 & 46.01 \\
\hline \multirow{2}{*}{ MEVi2 } & $\mathrm{OE}$ & \multirow{2}{*}{1.5} & \multirow{2}{*}{3.45} & $\begin{array}{c}88.3 \\
3\end{array}$ & \multirow{2}{*}{$\begin{array}{c}66.3 \\
8\end{array}$} & 0.02 & 6.79 \\
\hline & $\mathrm{R} 1$ & & & $\begin{array}{c}19.2 \\
4\end{array}$ & & 0.10 & 31.19 \\
\hline \multirow{2}{*}{ MEVi3 } & $\mathrm{OE}$ & \multirow{2}{*}{1.75} & \multirow{2}{*}{4.03} & $\begin{array}{c}119 . \\
8\end{array}$ & \multirow{2}{*}{$\begin{array}{c}107 . \\
3\end{array}$} & 0.02 & 5.01 \\
\hline & $\mathrm{R} 1$ & & & $\begin{array}{c}26.6 \\
5\end{array}$ & & 0.08 & 22.51 \\
\hline \multirow[b]{2}{*}{ MEVi4 } & $\mathrm{OE}$ & \multirow[b]{2}{*}{2} & \multirow[b]{2}{*}{4.60} & 156 & \multirow{2}{*}{$\begin{array}{c}162 . \\
2\end{array}$} & 0.01 & 3.85 \\
\hline & $\mathrm{R} 1$ & & & $\begin{array}{c}35.2 \\
5\end{array}$ & & 0.06 & 17.02 \\
\hline \multirow[b]{2}{*}{ MEVi5 } & $\mathrm{OE}$ & \multirow[b]{2}{*}{2.25} & \multirow[b]{2}{*}{5.18} & 197 & \multirow[b]{2}{*}{$\begin{array}{c}233 . \\
1\end{array}$} & 0.01 & 3.05 \\
\hline & $\mathrm{R} 1$ & & & $\begin{array}{c}45.0 \\
5\end{array}$ & & 0.04 & 13.32 \\
\hline \multirow{2}{*}{ MEVi6 } & $\mathrm{OE}$ & \multirow{2}{*}{2.5} & \multirow{2}{*}{5.75} & $\begin{array}{c}242 . \\
7\end{array}$ & \multirow{2}{*}{$\begin{array}{c}322 . \\
3\end{array}$} & 0.01 & 2.47 \\
\hline & $\mathrm{R} 1$ & & & $\begin{array}{c}56.0 \\
5\end{array}$ & & 0.04 & 10.70 \\
\hline
\end{tabular}

Tabla 3 Condiciones de frontera para realizar simulaciones

\section{Calibración y validación}

El procedimiento para realizar la calibración consistió en colocar los dispositivos por periodo de un día, como indica la siguiente Figura:

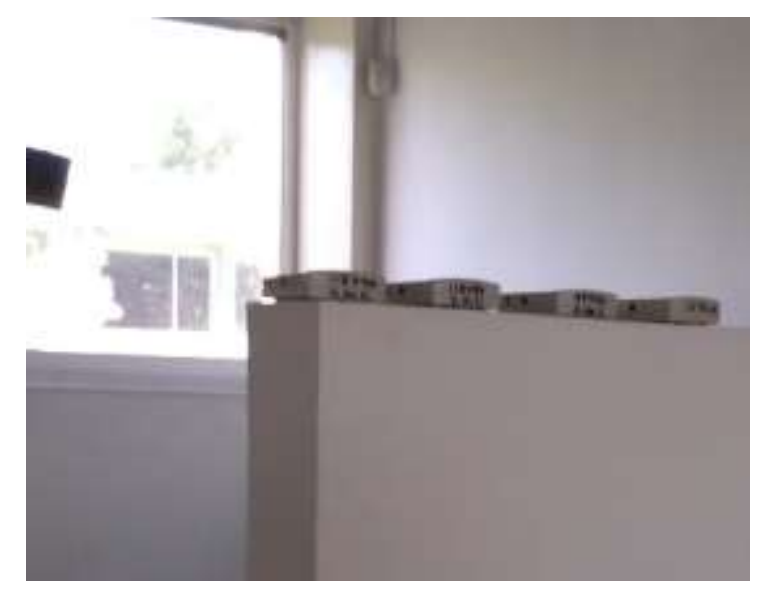

Figura 6 Localización de dispositivos para realizar la calibración

Se obtuvieron líneas de tendencia y ecuaciones de corrección a partir del establecimiento del dispositivo de referencia y los contrastes (Tabla 04).

\begin{tabular}{|c|c|c|c|c|c|c|c|}
\hline No. & \multicolumn{2}{|c}{$\begin{array}{c}\text { No. } \\
\text { Datos }\end{array}$} & \multicolumn{2}{c}{ Referencia } & \multicolumn{2}{c}{ Contraste } & \multicolumn{2}{c|}{$\mathbf{R}^{2}$} & \multicolumn{2}{c}{$\begin{array}{c}\text { Ecuación de } \\
\text { corrección }\end{array}$} & \multicolumn{1}{c|}{$\begin{array}{c}\text { Error } \\
\text { típico }\end{array}$} \\
\hline 1 & 50 & 1300808 & 1300805 & 0.9998 & $y=0.9772 x+0.7742 .02$ & 0.029 \\
\hline 2 & 50 & 1300808 & 1300804 & 0.9998 & $v=1.027 x-0.7365$ & 1.97 & 0.028 \\
\hline 3 & 50 & 1300808 & 1016364 & 0.9998 & $=0.9759 x+0.89362 .02$ & 0.028 \\
\hline
\end{tabular}

Tabla 4 Resultados obtenidos de acuerdo a la calibración realizada.

Fuente: (Esparza López, 2015) 
Se capturaron 50 datos, que corresponden a la variable Temperatura de bulbo seco, utilizando data logger modelo U12. Las ecuaciones obtenidas, permiten corregir errores sistemáticos de los dispositivos de medición, estos datos de calibración definen las ecuaciones finales de corrección.

\section{Validación}

La validación se realizó en una vivienda ubicada en la localidad El Salitre, San José Iturbide Guanajuato, se analizaron 8 prototipos de vivienda más utilizados en el Estado de Guanajuato de acuerdo a información de INFONAVIT delegación Guanajuato. El modelo seleccionado enriquece la condición paramétrica para vincular el diseño de la dimensión del espacio con la clasificación arquitectónica y la clasificación climática. Kaplan y Canner recomendaron para simulaciones energéticas como satisfactoria la diferencia de 5\% (mensual) y de $15 \%$ (diaria). La diferencia aceptable puede aumentar hasta un $15-25 \%$ (mensual) y $25-35 \%$ (diario) para la simulación de sistemas HVAC. El uso anual de energía simulada debe estar dentro del 10\% de la información recopilada.

Por otra parte, (Ali \& Abustan, 2014) proponen el Porcentaje Medio de Error Relativo Absoluto (PMARE) que se muestra en la ecuación 1.

$\operatorname{PMARE}(\%)=\frac{100}{n} \sum_{i=1}^{n} \frac{\operatorname{Abs}\left(O_{i}-P_{i}\right)}{O_{i}}$

Dónde:

$O_{i}=$ Valores observados

$P_{i}=$ Valores simulados

$\mathrm{Abs}=$ Valor absoluto (de la diferencia entre el valor observado y valor simulado

Ecuación 1 Porcentaje Medio de Error Relativo Absoluto (Ali \& Abustan, 2014)

El periodo de validación fue del 26 de octubre al 02 de noviembre del 2018. Se observó la temperatura de bulbo seco con base horario, la ubicación de los dispositivos se determinó al centro geométrico de cada espacio, a una altura de $0.1 \mathrm{~m}, 0.6 \mathrm{~m}$ y $1.1 \mathrm{~m}$ como lo establece ASHRAE Standard 55-2010.
ASTM C-1046-95 indica que se deben evitar las ganancias o pérdidas por infiltración, sin embargo, la vivienda que se utilizó para esta investigación es propiedad privada, no se pudieron sellar puertas y ventanas. Los datos medidos corresponden a la semana de monitoreo en la unidad de estudio, los datos simulados corresponden a los resultados obtenidos en Design Builder. De acuerdo a la clasificación del rendimiento del modelo de evaluación (Ali \& Abustan, 2014), los resultados son aceptables (Tabla 05).

\begin{tabular}{|c|c|c|c|c|}
\hline \multirow{2}{*}{\multicolumn{5}{|c|}{$\begin{array}{c}\text { Temperatura media de bulbo seco (Tml } \\
\text { El Salitre, SJI Recámara } 1(\mathrm{R} 1) \\
\text { Periodo A }\end{array}$}} \\
\hline & & & & \\
\hline \multirow[b]{2}{*}{ Mes } & \multirow[b]{2}{*}{ Día } & \multirow[b]{2}{*}{$\begin{array}{c}\text { Medido } \\
\text { promedio } \\
\cap .\end{array}$} & simulado & PMARE \\
\hline & & & $P_{i}$ & $(\%)=\frac{100}{n} \sum_{i=1}^{n} \frac{A b s\left(O_{i}-P_{i}\right)}{O_{i}}$ \\
\hline \multirow[t]{6}{*}{ Octubre } & Vie 26 & 23.09 & 20.19 & 0.13 \\
\hline & Sab 27 & 21.90 & 20.87 & 0.05 \\
\hline & Dom 28 & 19.96 & 22.33 & 0.12 \\
\hline & $\mathrm{Lu} 29$ & 19.65 & 22.39 & 0.14 \\
\hline & Mar 30 & 20.58 & 23.43 & 0.14 \\
\hline & Mie 31 & 21.80 & 24.53 & 0.12 \\
\hline \multirow[t]{2}{*}{ Noviembre } & Jue 01 & 21.41 & 20.70 & 0.03 \\
\hline & Vie 02 & 19.99 & 22.73 & 0.14 \\
\hline & & & PMARE & 10.80 \\
\hline
\end{tabular}

Tabla 5 Resultados de validación

\section{Resultados}

Una vez realizadas las simulaciones, el cálculo de la potencia se realizó con base en la siguiente ecuación:

Potencia $=\left[\rho C_{\rho} V(\right.$ Tconfort - Tsimulación $\left.)\right] / 3600 s$

Para obtener la demanda de energía, se agruparon los datos de acuerdo a la tarea de enfriamiento o tarea de calentamiento por mes con base horario, obteniendo resultados en $\mathrm{kWh}$ como lo indican los siguientes gráficos: 


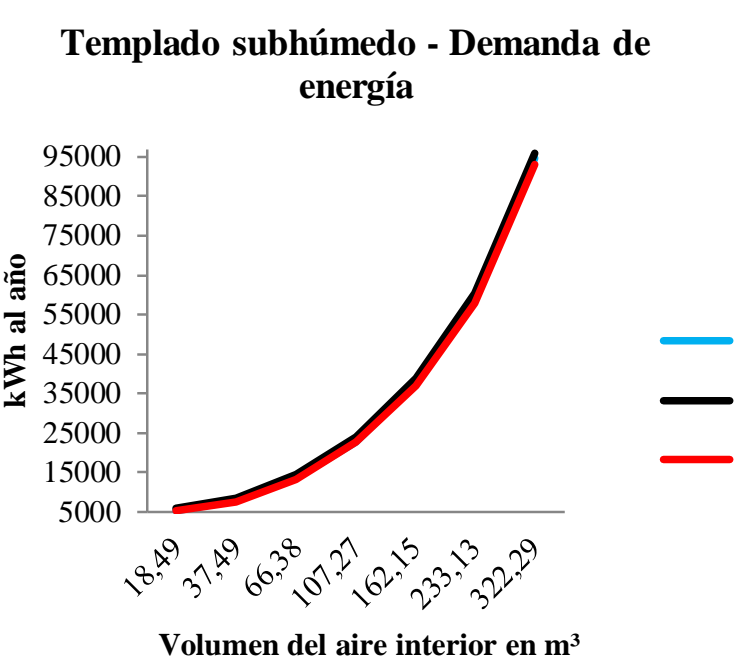

Gráfico 1 Demanda de energía para Templado sub húmedo en $\mathrm{kWh}$. Se realizaron tres operaciones: ventana abierta, ventana cerrada y operación selectiva

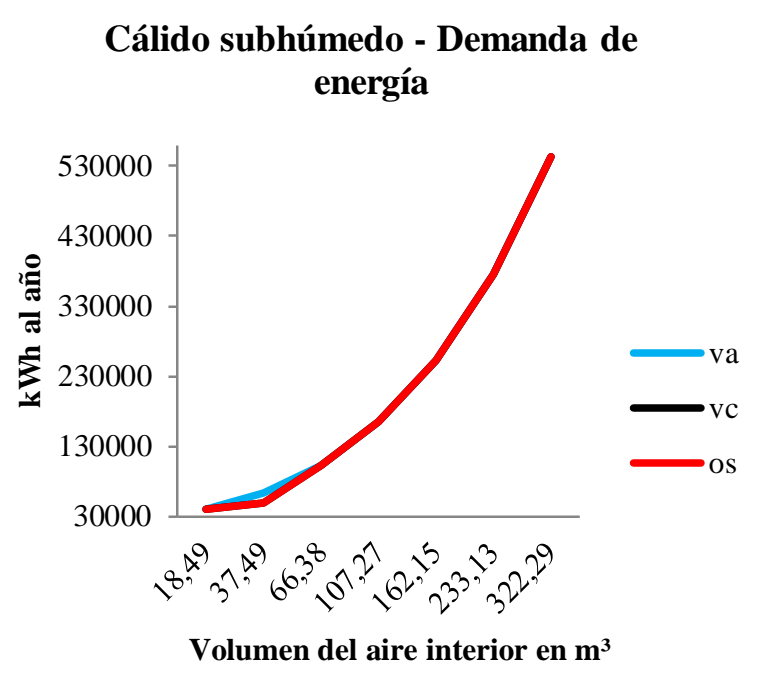

Gráfico 2 Demanda de energía para Cálido sub húmedo en $\mathrm{kWh}$. Se realizaron tres operaciones: ventana abierta, ventana cerrada y operación selectiva

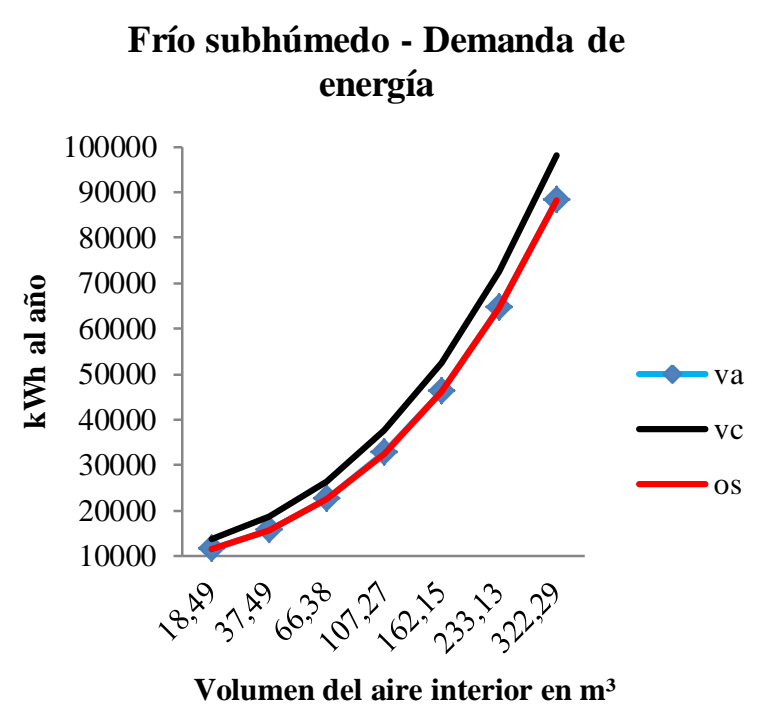

Gráfico 3 Demanda de energía para Frío sub húmedo en $\mathrm{kWh}$. Se realizaron tres operaciones: ventana abierta, ventana cerrada y operación selectiva

\section{Análisis de resultados}

Los resultados muestran que al incrementar el volumen de aire interior, aumentó también la demanda de energía en cada condición térmica, y mostró una tendencia a mantener ventana abierta, al realizar la operación selectiva, en todos los modelos simulados y en las tres concidiones térmicas establecidas. El cálido sub húmedo muestra un punto de discrepancia entre las tres operaciones (ventanas cerradas, ventanas abiertas y operación selectiva) en el volumen de aire interior $37.49 \mathrm{~m}^{3}$, que corresponde al MEVi1.

A partir de este punto, se observa una separación entre mantener ventana abierta y operación selectiva contra mantener ventana cerrada. Para el caso del templado sub húmedo, se observa un comportamiento similar que muestra una diferencia menor entre mantener ventana cerrada contra mantener ventana abierta y la operación selectiva.

En el frío sub húmedo se observó una separación entre mantener ventanas abiertas y operación selectiva contra mantener ventanas cerradas en el MEVi1, al incrementar el volumen de aire interior, aumenta esta separación.

Para comprender la demanda de energía en términos de las diferentes condiciones térmicas que se presentan en el estado de Guanajuato, México, se realizó el análisis de las tareas de control, calentamiento y enfriamiento.

En términos de las tareas de confort, calentamiento y enfriamiento, se determinó un punto de intersección en el MEVi3 (107.27 $\left.\mathrm{m}^{2}\right)$ para el templado sub húmedo (Gráfico 04), disminuye la tarea de enfriamiento pero aumenta la tarea de calentamiento, por lo que se observa una tendencia a mantener un volumen intermedio entre los modelos observados. 
Horas-tarea templado subhúmedo

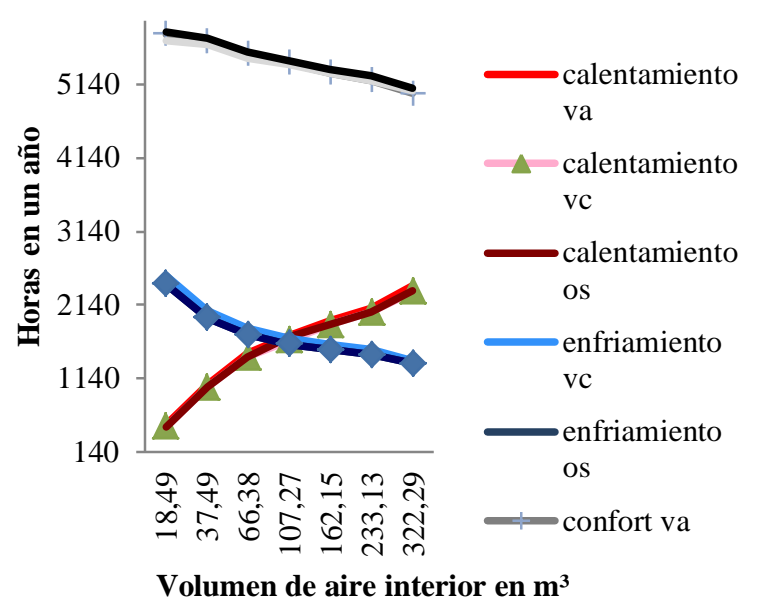

Gráfico 4 Tarea de confort, enfriamiento y calentamiento para el templado sub-húmedo

En el cálido sub-húmedo predomina la tarea de enfriamiento, observándose un punto de coincidencia en el MEVil $\left(37.49 \mathrm{~m}^{2}\right)$ (Gráfico 05). En el frío sub-húmedo se observa el mayor número de horas de confort en el MEVi1 $\left(37.49 \mathrm{~m}^{2}\right)$. Al aumentar el volumen de aire interior, disminuyen las horas de confort, lo que determina una tendencia por espacios reducidos en esta condición térmica (Gráfico $06)$.

\section{Horas-tarea cálido subhúmedo}

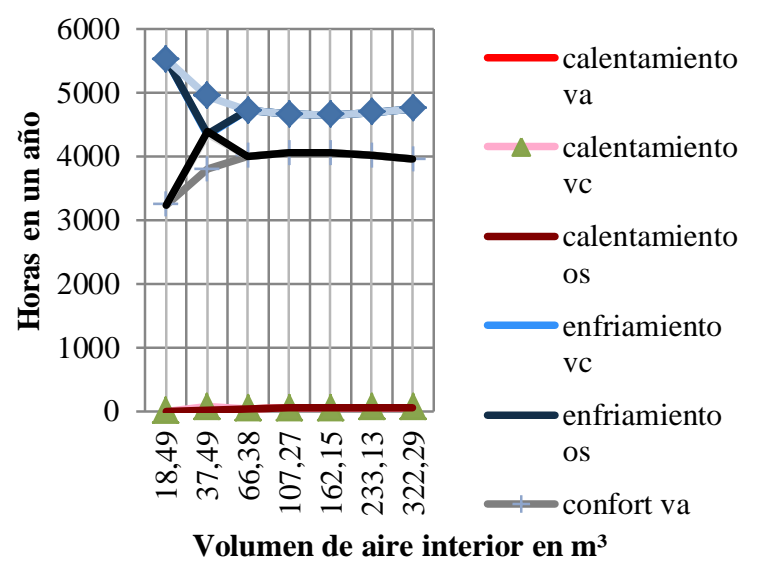

Gráfico 5 Tarea de confort, enfriamiento y calentamiento para el cálido sub-húmedo

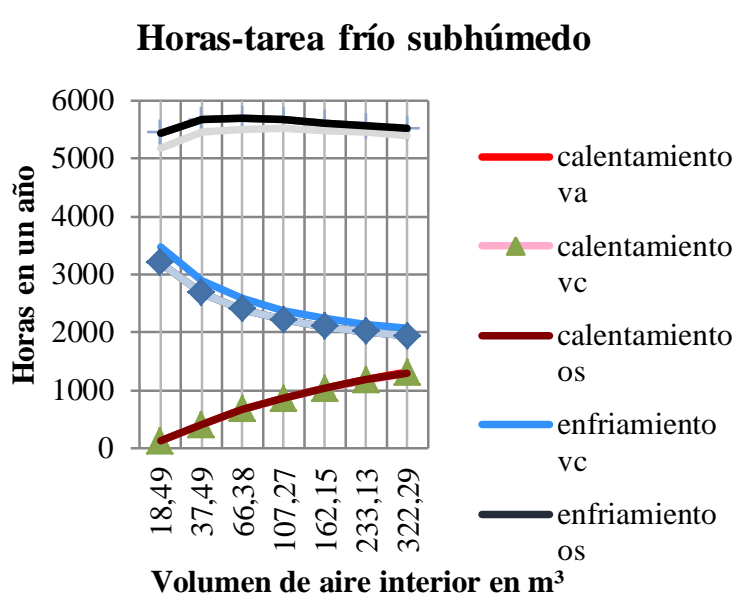

Gráfico 6 Tarea de confort, enfriamiento y calentamiento para el frío sub-húmedo

\section{Agradecimientos}

La presente investigación se ha realizado gracias a la beca otorgada por el Consejo Nacional de Ciencia y Tecnología (Conacyt).

\section{Conclusiones}

Se identificó la demanda energética anual, en diferentes condiciones volumétricas y diferentes condiciones térmicas del estado de Guanajuato, con un rango de confort establecido. Se definió, en el escenario virtual, la relación directamente proporcional entre volumen del aire interior y demanda energética en el espacio representativo (Recámara 1), por cada condición térmica. Se determinaron periodos con base horario de apertura o cierre de ventana en función del rango de confort establecido para cada localidad. Se comprobó que en las tres condiciones térmicas, al aumentar el volumen de aire interior, aumentó la demanda energética, y en términos de tareas de confort, enfriamiento y calentamiento, se determinó que: Para el templado sub-húmedo se recomienda el MEVi3 $\left(107.27 \mathrm{~m}^{2}\right)$, a partir de este volumen, la tarea de enfriamiento disminuye pero aumenta considerablemente la tarea de calentamiento. Para el cálido subhúmedo, se recomienda el MEVi1 $\left(37.49 \mathrm{~m}^{2}\right)$, debido al aumento de la tarea de confort y disminución de la tarea de enfriamiento en un punto de coincidencia. Y finalmente, se recomienda para el frío sub-húmedo, el MEVi1 $\left(37.49 \mathrm{~m}^{2}\right)$. En este caso, se observó una tendencia a mantener un menor volumen de aire interior, para lograr un mayor número de horas de confort por año. 
Se concluye que las condiciones térmicas, y el volumen de aire interior, son elementos que determinan la demanda energética en función del confort térmico, cuando se utiliza ventilación modo mixto. Se recomienda, para futuras investigaciones, abarcar otras condiciones térmicas y analizar en términos del equipo de aire acondicionado, estrategias de ahorro energético.

\section{Referencias}

Ali, M., \& Abustan, I. (2014). A new novel index for evaluating model performance. Malasia: Jornal of Natural Resources and Development.

ASHRAE. (2004). American Society of Heating, Refrigerating and Air-Conditioning Engineers. Atlanta: ASHRAE. Recuperado el 06 de JUNIO de 2017

Ashrae, S.-2. (2010). Thermal Environmental Conditions for human occupancy. Atlanta: Ashrae.

Barrios, G., Huelsz, G., Rechtman, R., \& Rojas, J. (2012). Wall/Roof Thermal performance differences between air-conditioned and non air-conditioned rooms. Centro de Investigación en Energía, 17.

Braulio, M., Bovea, M., Rua, M., \& Juan, P. (2016). A methodology for predicting the energy performance and indoor thermal comfort of residential stocks on the neighbourhood and city scales. A case study in Spain. ScienceDirect.

Carballo Méndez, M. G. (2017). Volumen óptimo de aire de un espacio habitable con el uso mixto de la ventilación natural y el aire acondicionado. Caso: clima cálido subhúmedo. Colima: Universidad de Colima.

Carrazco Cota, C. (2005). Análisis cuantitativo de comportamiento térmico de los elementos bioclimáticos de la arquitectura vernácula: caso clima cálido seco. Distrito Federal: UNAM.

Casas Arredondo, J. M. (2014). Simulación térmica de edificaciones con EnergyPlus y Ener-Habitat: análisis de la interacción entre los componentes de la envolvente. Temixco, Morelos.: Universidad Nacional Autónoma de México.
Comisión Nacional de Vivienda. (2017). Reporte mensual del sector de la vivienda. Ciudad de México: CONAVI.

CONAVI. (03 de 07 de 2019). Comisión Nacional de Vivienda. Obtenido de http://sniiv.conavi.gob.mx/

CONUEE. (2017). Comisión Nacional para el Uso Eficiente de la Energía. Recuperado el 07 de junio de 2017, de Documentos: https://www.gob.mx/conuee\#documentos

Deuble, M., \& John de Dear, R. (2012). Mixedmode buildings: a double standard in occupants'comfort expectations. Australia: Elsevier.

Esparza López, C. J. (2015). Estudio Experimental de dispositivos de enfriamiento evaporativo indirecto paa un clima cálido subhúmedo. Colima: Programa Interinstitucional de Doctorado en Arquitectura.

Ezzeldin, S., \& Rees, S. (2013). The potential for office buildings with mixed-mode ventilation and low energy coolings systems in arid climates. ScienceDirect.

Gómez Amador, A., Espinoza López, A. E., Bojórquez Morales, G., \& Alpuche Cruz, M. G. (2015). Mecanismos de control bioclimáticos habilitados por los individuos en viviendas de climas cálidos. En A. Gómez Amador, A. E. Espinoza López, G. Bojórquez Morales, \& M. G. Alpuche Cruz, Diversas visiones de la habitabilidad (pág. 251). Puebla: Programa Editorial de la Red de Investigación Urbana A.C.

Gto., D. U. (03 de julio de 2019). León Gobierno municipal. Obtenido de http://du.leon.gob.mx/contenidos/leyes-yreglamentos

Gto., S. F. (03 de julio de 2019). San Felipe. Obtenido de http://sanfelipegto.gob.mx/TRANSPARENCIA /I\%20-\%20REGLAMENTOS.html

Gto., S. J. (03 de 07 de 2019). San José Iturbide municipio. Obtenido de http://normatividadestatalymunicipal.guanajuat o.gob. $\mathrm{mx} /$ normatividadn/archivos.php? arcid=9 6

VÁZQUEZ-TORRES, Claudia Eréndira, GÓMEZ-AMADOR, Adolfo y ESCOBAR-DEL POZO, Carlos. Desempeño térmico de un espacio habitable con ventilación modo mixto. Diferentes condiciones volumétricas y diferentes condiciones térmico ambientales en el Estado de Guanajuato. México. Revista de Arquitectura y Diseño. 2019 
Gto., S. J. (03 de 07 de 2019). San José Iturbide municipio. Obtenido de http://normatividadestatalymunicipal.guanajuat o.gob.mx/normatividadn/archivos.php?arcid=9 6

INEGI. (2017). Instituto Nacional de Estadística y Geografía. Recuperado el 04 de junio de 2017, de http://www.beta.inegi.org.mx/app/biblioteca/fic ha.html?upc=702825292775

INFONAVIT. (2017). Instituto del Fondo Nacional de la Vivienda para los Trabajadores. Recuperado el 05 de junio de 2017, de https://portal.infonavit.org. $\mathrm{mx} / \mathrm{wps} / \mathrm{wcm} /$ conne ct/e3d15d95-f875-45e4-8772-

9078660eca81/Anexo+1.+Listado+de+regiones +bioclim\%C3\%A1ticas.pdf?MOD=AJPERES $\& C O N V E R T \_T O=u r l \& C A C H E I D=e 3 d 15 d 95-$ f875-45e4-8772-9078660eca81

Monfet, D., Zmeureanu, R., Charneux, R., \& Lemire, N. (2007). Computer model of a university building using the EnergyPlus program. Quebec: Concordia University.

ONNCCE. (2009). NMX-C-460-ONNCCE2009. MÉXICO: ONNCCE.

S., E., \& Rees, S. (2013). The potential for office buildings with mixed-mode ventilation and low energy cooling systems in arid climates. Elsevier.

SENER. (2011). Indicadores de Eficiencia Energética en México. 5 sectores, 5 retos. México, D. F.: SENER.

Szokolay, S. (2004). Introduction to ARCHITECTURAL SCIENCE the basis of sustainable design. Gran Bretaña: Elsevier. 